\title{
Direct Visualization of Anti-Ferroelectric Switching Dynamics via Electrocaloric Imaging
}

\author{
Pablo Vales-Castro,* Miquel Vellvehi, Xavier Perpiñà, Jose Manuel Caicedo, \\ Xavier Jordà, Romain Faye, Krystian Roleder, Dariusz Kajewski, Amador Perez-Tomas, \\ Emmanuel Defay, and Gustau Catalan*
}

The large electrocaloric coupling in $\mathrm{PbZrO}_{3}$ allows using high-speed infrared imaging for visualizing anti-ferroelectric switching dynamics via the associated temperature change. It is found that in ceramic samples of homogeneous temperature and thickness, switching is fast due to the generation of multiple nucleation sites, with devices responding in the millisecond range. By introducing gradients of thickness, however, it is possible to change the dynamics to propagation limited, whereby a single-phase boundary sweeps across the sample like a cold front, at a speed of $\approx 20 \mathrm{~cm} \mathrm{~s}^{-1}$. Additionally, introducing thermostatic temperature differences between two sides of the sample enables the simultaneous generation of a negative electrocaloric effect on one side and a positive one on the other, yielding a Janus-like electrocaloric response. or even the standard gas cooling cycle (50\%). ${ }^{[1]}$ First theorized in 1878 by William Thomson, ${ }^{[2]}$ the high-temperature changes $(\Delta T=12 \mathrm{~K})$ calculated for ferroelectric $(\mathrm{FE})$ thin films ${ }^{[3]}$ and the discovery of an anomalous electrocaloric effect in anti-ferroelectrics $(\mathrm{AFE})^{[3]}$ have renewed its interest, with an eye put on its potential application as a solid-state cooling solution in integrated circuits.

FE materials display what is regarded as the "conventional" electrocaloric effect (or positive electrocaloric effect), whereby the material increases temperature $(\Delta T>0)$ when a voltage step is applied and decreases temperature $(\Delta T<0)$ when it is removed. In contrast, AFE display the

\section{Introduction}

The electrocaloric effect (ECE) consists of a transient temperature change $(\Delta T)$ when a voltage step is applied adiabatically to a dielectric sample. It has a high theoretical efficiency $(70 \%)$, higher than solid-state analogs like thermoelectrics (20\%),

P. Vales-Castro, J. M. Caicedo, A. Perez-Tomas, G. Catalan

Catalan Institute of Nanoscience and Nanotechnology (ICN2)

Campus Universitat Autonoma de Barcelona

Av. Serragalliners s/n, Bellaterra 08193, Spain

E-mail: pablo.vales@icn2.cat; gustau.catalan@icn2.cat

M. Vellvehi, X. Perpiñà, X. Jordà

Institut de Microelectrònica de Barcelona (IMB-CNM,CSIC)

Carrer dels Til.lers $s / n$

Campus Universitat Autònoma de Barcelona (UAB)

Cerdanyola del Vallès 08193, Spain

R. Faye, E. Defay

Materials Research and Technology Department

Luxembourg Institute of Science and Technology

University of Luxembourg

2 Avenue de l'Université, Esch-sur-Alzette L-4365, Luxembourg

K. Roleder, D. Kajewski

Institute of Physics

University of Silesia in Katowice

ul. Uniwersytecka 4, Katowice 40-00, Poland

G. Catalan

Institut Català de Recerca i Estudis Avançats (ICREA)

Barcelona, Catalunya 08010, Spain

The ORCID identification number(s) for the author(s) of this article can be found under https://doi.org/10.1002/aelm.202100380.

DOI: 10.1002/aelm.202100380 opposite response; they decrease temperature $(\Delta T<0)$ when an electric field $E$ is applied, and increase $(\Delta T>0)$ when it is removed. The ability of anti-ferroelectrics to cool down despite electrostatic energy being pumped into them is intriguing, and different underlying mechanisms have been proposed for the negative electrocaloric effect. ${ }^{[4,5]}$ Recent experimental evidence indicates that, in the archetypal anti-ferroelectric $\mathrm{PbZrO}_{3}$ (PZO), the so-called giant negative electrocaloric effect is due to the latent heat absorbed during the adiabatic-field-induced AFE-FE transition, which is endothermic. ${ }^{[6]}$

In $\mathrm{PbZrO}_{3}$, the direct link between the anomalous electrocaloric effect and the first-order anti-ferroelectric-ferroelectric switching implies that the field-induced nucleation and motion of the AFE-FE phase boundary will dictate the dynamics of the large negative ECE and, ultimately, the dynamics of electrocaloric devices based on first-order transitions. In ferroelectrics, the study of domain wall dynamics ${ }^{[7-16]}$ has been examined in detail on account of their relevance for ferroelectric memories. In contrast, there are far fewer works regarding the dynamics of the ferroelectric-paraelectric phase boundaries in $\mathrm{FE}^{[17,18]}$ or the anti-ferroelectric-ferroelectric ones in anti-ferroelectrics. ${ }^{[19-23]}$ Yet, a priori, one cannot assume that the dynamics of domain walls will be the same as the dynamics of phase boundaries, while the former separate different domains within the same ferroelectric phase, the latter separate different phases-in antiferroelectrics, an antipolar phase from a field-induced polar one.

Domain switching dynamics is defined by a nucleationpropagation process. On the one hand, nucleation refers to the appearance of "hotspots," where nanoscopic nuclei of switched domains appear and rapidly expand forward across the thickness 
of the sample. ${ }^{[13]}$ This process of nucleation and forward growth is known to be very fast; the nucleation points appear in a time $t_{\mathrm{n}} \approx 1 \mathrm{~ns}$ while the forward growth can be usually calculated like $t \sim \frac{d}{v_{\text {sound }}}$ where $d$ and $v_{\text {sound }}$ are the sample thickness and the sound velocity in the material, respectively. ${ }^{[13]}$ This implies that the complete formation of nucleation points in an $\approx 100 \mu \mathrm{m}$ thick sample would take $\approx 100$ ns (assuming $v_{\text {sound }} \approx$ $10^{3} \mathrm{~m} \mathrm{~s}^{-1}$ ). On the other hand, propagation refers to the sideways' expansion of such nucleated domains along the rest of the sample until the whole volume has switched to the final phase. This sideways' growth time $t_{\mathrm{s}}$ can be slow, and it typically varies as a function of electric field like $t_{\mathrm{s}}=\alpha E^{-\frac{3}{2},{ }^{, 13]}}$ where $\alpha$ is a constant.

Despite their difference, nucleation and propagation always take place in a switching process. However, one can be dominant with respect to the other. Therefore, the switching mechanisms can be divided into two kinds: nucleation limited and propagation limited. In the former, switching is dominated by nucleation, that is, the sample is mostly switched by the appearance of a large number of nucleation points (and thus switching is very fast), while in the latter, switching is dominated by sideways propagation, which implies a low amount of nucleation points that expand across the sample (and thus switching is slower).

Besides its fundamental interest, the study of switching dynamics in anti-ferroelectrics is important for determining the response speed of anti-ferroelectric devices, in general, and electrocaloric ones, in particular. However, it is often an overlooked topic, partly due to the technical difficulty of implementing a direct and reliable spatially resolved technique (like infrared (IR) imaging) with the high sampling rate needed to reliably characterize it. Only a few works have reported timeand space-resolved infrared frames during an electrocaloric cycle, ${ }^{[24,25]}$ and none of them focused on the mechanism or the dynamics of the electrocaloric responses, but rather on the absolute $\Delta T$. In 2016, Liu et al. ${ }^{[26]}$ reported a spatially resolved study of the heat flux across a multilayer capacitor that provided hints for an efficient way of fabricating multilayer capacitors for an optimal heat exchange, but no further works were done in this aspect.
It is therefore the aim of this work to visualize and quantify the dynamics of the AFE-FE phase transition, determine its dominant mechanism (i.e., nucleation limited or propagation limited), and measure the speed of the anti-ferroelectric phase boundary.

\section{Results and Discussion}

The samples examined in this study are ceramics of pure PZO, an archetypal anti-ferroelectric with a large negative electrocaloric effect. ${ }^{[5,27,28]}$ Thanks to the electrocaloric temperature change concomitant with the AFE-FE phase transition (with $\mathrm{Pbam}^{[29]}$ and $R 3 c^{[30]}$ symmetries, respectively), it is possible to use an infrared camera to observe how the electrocaloric front linked to the AFE switching nucleates/propagates across the sample in real time at a maximum frequency of $1253 \mathrm{~Hz}$. In order to be able to switch the bulk ceramic capacitors with electric fields lower than the breakdown field, we work at temperatures close to, but below, the Curie temperature, which for $\mathrm{PZO}$ is $T_{\mathrm{C}} \approx 230{ }^{\circ} \mathrm{C}$. The electrocaloric response and switching dynamics are shown in Figure 1.

For a given electric field, the speed of the AFE-FE phase transition (and thus the speed of the negative ECE) increases with the sample's temperature (Figure 1). This increasing switching speed is a consequence of the higher thermal energy available to promote this transition, which weakens the anti-ferroelectric coupling and decreases the critical switching field as temperature approaches $T_{\mathrm{c}}$. A direct consequence is that, for a given temperature, the ability of a field to switch the anti-ferroelectric depends on how much time the field is applied for. In Figure $1 \mathrm{~b}$ we see, for example, that while at $214^{\circ} \mathrm{C}$ switching happens in $10 \mathrm{~ms}$ for the applied field of $35 \mathrm{kV} \mathrm{cm}{ }^{-1}$, at $204{ }^{\circ} \mathrm{C}$ the same field needs to be applied for more than $25 \mathrm{~ms}$ for switching to happen. This behavior is reminiscent of the frequency dependence of the coercive field in ferroelectric hysteresis. ${ }^{[13]}$

For the fastest electrocaloric response (high field at high temperature), the switching is so fast that even at the fastest available frame rate of $1250 \mathrm{fps}$, most of the change happens within only a couple of frames (Figure 2). Nevertheless, there is still enough information to quantify the switching speed (a)

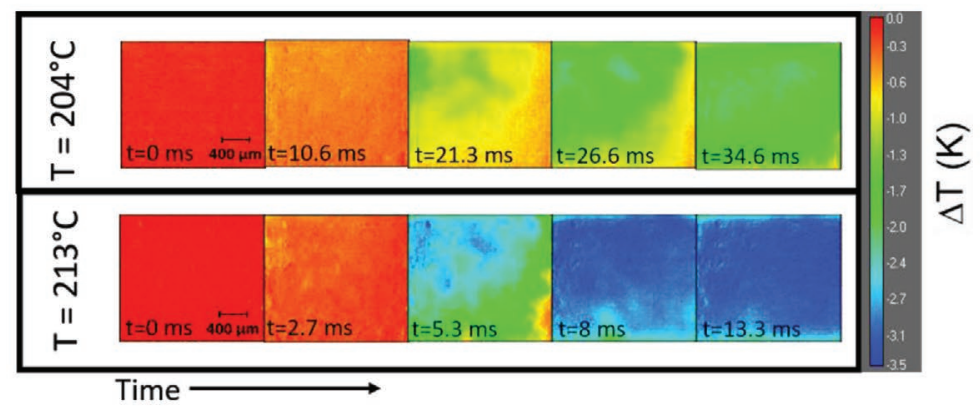

(b)

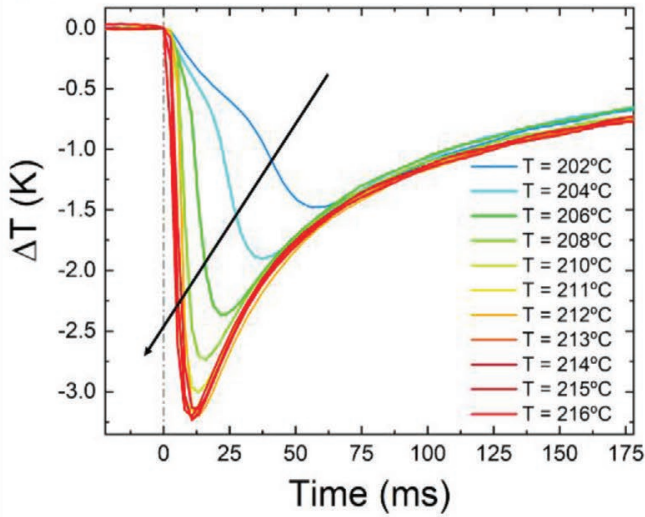

Figure 1. a) Anti-ferroelectric-ferroelectric switching dynamics observed by infrared imaging at two different temperatures for an electric field $E=35 \mathrm{kV} \mathrm{cm}^{-1}$ and a sampling frequency of $376 \mathrm{~Hz}$. Note that time labels for both temperatures differ. Each frame corresponds to $1 \mathrm{~mm}^{2}$ area. b) Electrocaloric profile as a function of time. It can be clearly seen that, as the temperature increases, the switching time decreases. 


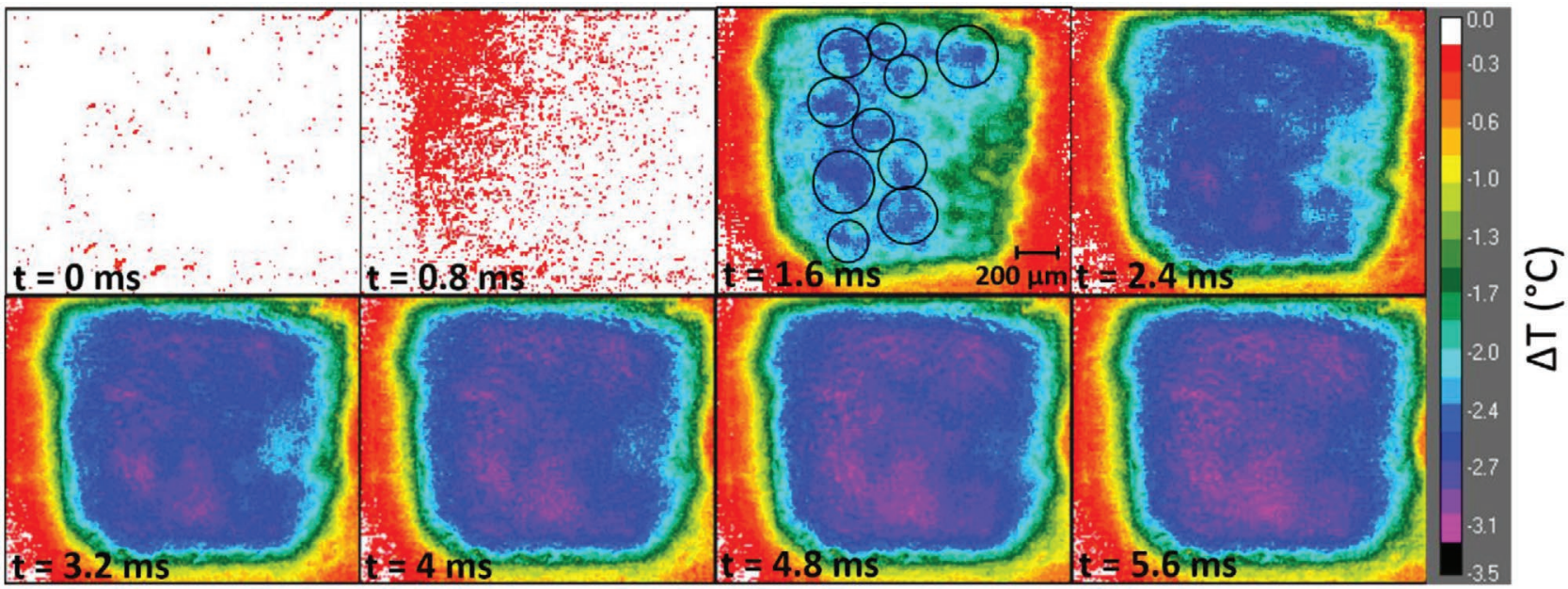

Figure 2. Infrared imaging of the large negative electrocaloric effect at $T=216 \mathrm{C}$ and $E=28 \mathrm{kV} \mathrm{cm}^{-1}$, acquired at a sampling rate of $1.25 \mathrm{kHz}$. Each frame has dimensions of $1.60 \mathrm{~mm} \times 1.28 \mathrm{~mm}$. The black circles represent the main nucleation points. From these points, the cold front propagates to cover the whole sample. Note: Pt electrodes do not reach the edges of the sample (to avoid air breakdown between the capacitor faces). This is the reason of the $\Delta T$ degradation at the edges, as no effective electric field is being applied there.

and address the question of whether switching is dominated by nucleation or by sideways propagation. The IR pattern immediately after switching is consistent with multiple-site nucleation; we can observe a mottled seed pattern distributed across the sample (Figure 2). The main nucleation centers (marked with black circles) can be distinguished as cold spots $(\Delta T \approx-2.5 \mathrm{~K})$. From frame 2 to frame 3 in Figure 2, we can see that the whole sample already displays a negative ECE $(\overline{\Delta T} \approx-2.2 \mathrm{C})$, which implies that the nucleation and sideways propagation process occur in time $t \ll 800 \mu$ s while the complete switching of the sample occurs in $\Delta t_{\mathrm{EC}} \approx 7.9 \mathrm{~ms}$. Given that the maximum averaged negative electrocaloric effect is $\Delta T=-3.2 \mathrm{~K}$, the electrocaloric response time $\left(\tau_{\mathrm{EC}}\right)$ is $\tau_{E C} \equiv \frac{\Delta t_{\text {max }}^{\max }}{\Delta T_{\max }} \approx 2.5 \mathrm{~ms} \mathrm{~K}^{-1}$. It may be worth noting that, although we can observe multiple nucleation sites, these are already developed nuclei that have undergone a subsequent sideways propagation. Therefore, given our time resolution, it is not possible to accurately discern if the process is really nucleation limited, i.e., the switching is mostly controlled by nucleation or, rather, a process where several nuclei appear but the switching is still dominated by propagation.

Nevertheless, it is clear that the appearance of multiple nuclei across the sample makes the switching fast but complicates the quantification of the phase boundary's sideways propagation velocity given the limits of the infrared camera's acquisition speed. In order to obtain a single nucleation point, and thus be able to observe the boundary's movement in a propagation-limited process, we have modified the morphology of the sample to give it a wedged profile (see the scheme in Figure 3b). In this way, the nucleation process starts at the thinnest side, where the electric field is more intense and propagates sideways from there toward the thicker side. The sample was polished with a thickness gradient $(\Delta d / \Delta x)$ of $16 \mu \mathrm{m} \mathrm{mm}^{-1}$ (75 $\mu \mathrm{m}$ difference across a $4.7 \mathrm{~mm}$ long sample with an average thickness of $160 \mu \mathrm{m})$. The resulting switching process was filmed, and the frames are shown in Figure 3. As expected, the phase transition starts at the thinner side of the wedge and then sweeps across the sample like a cold "weather front."
For the largest applied field of $35 \mathrm{kV} \mathrm{cm}^{-1}$, the front propagates sideways with a maximum velocity $v=20 \mathrm{~cm} \mathrm{~s}^{-1}$, resulting in a device electrocaloric response time $\tau_{E C}=\frac{\Delta_{t}^{\max }}{\Delta T_{\max }}=9.8 \mathrm{~ms} \mathrm{~K}^{-1}$. For $E=28 \mathrm{kV} \mathrm{cm}^{-1}$, which is the same field used for the homogeneous thickness sample in Figure 2, the electrocaloric response time is $\tau_{E C}=\frac{\Delta t_{T C}^{\max }}{\Delta T_{\max }}=12 \mathrm{~ms} \mathrm{~K}^{-1}$. The response time of the propagation-limited dynamics (wedge) is therefore 4-5 times slower than that of the thickness homogeneous parallelplate capacitor.

The results demonstrate that thickness gradients can be used to modulate the dynamics of the electrocaloric effect. Thickness, however, is not the only magnitude that can be graded; gradients of temperature can also be used. For example, by sitting the sample at the edge of the heater, a temperature step can be introduced, thereby forcing the sample to bridge across two different points of its phase diagram. Since PZO has both an AFE-FE transition with the negative electrocaloric effect and a ferroelectric-paraelectric one with a positive ECE (Figure 4), bridging the two transitions enables the possibility of a "Janus electrocaloric effect:" a simultaneous positive and negative electrocaloric response upon applying a voltage to the sample (Figure 4). It is worth noting that, although our setup technical constraints did not allow us, this effect can be easily maximized by controlling the temperature of both halves of the sample with two independent Peltiers; this would generate an extreme "Janus electrocaloric effect" whereby one side would display $\Delta T \approx-3.2{ }^{\circ} \mathrm{C}$ and the other $\Delta T \approx+5.5^{\circ} \mathrm{C}$, and thus a thermal difference is of almost $9{ }^{\circ} \mathrm{C}$.

\section{Conclusions}

The strong locking between anti-ferroelectric switching and electrocaloric response thus enables the observation in real time of the switching dynamics and its manipulation by playing with the temperature and/or morphology of the sample. The switching in homogeneous anti-ferroelectric capacitors of $\approx 150 \mu \mathrm{m}$ is fast due to the generation of multiple nucleation 
(a)

(b)
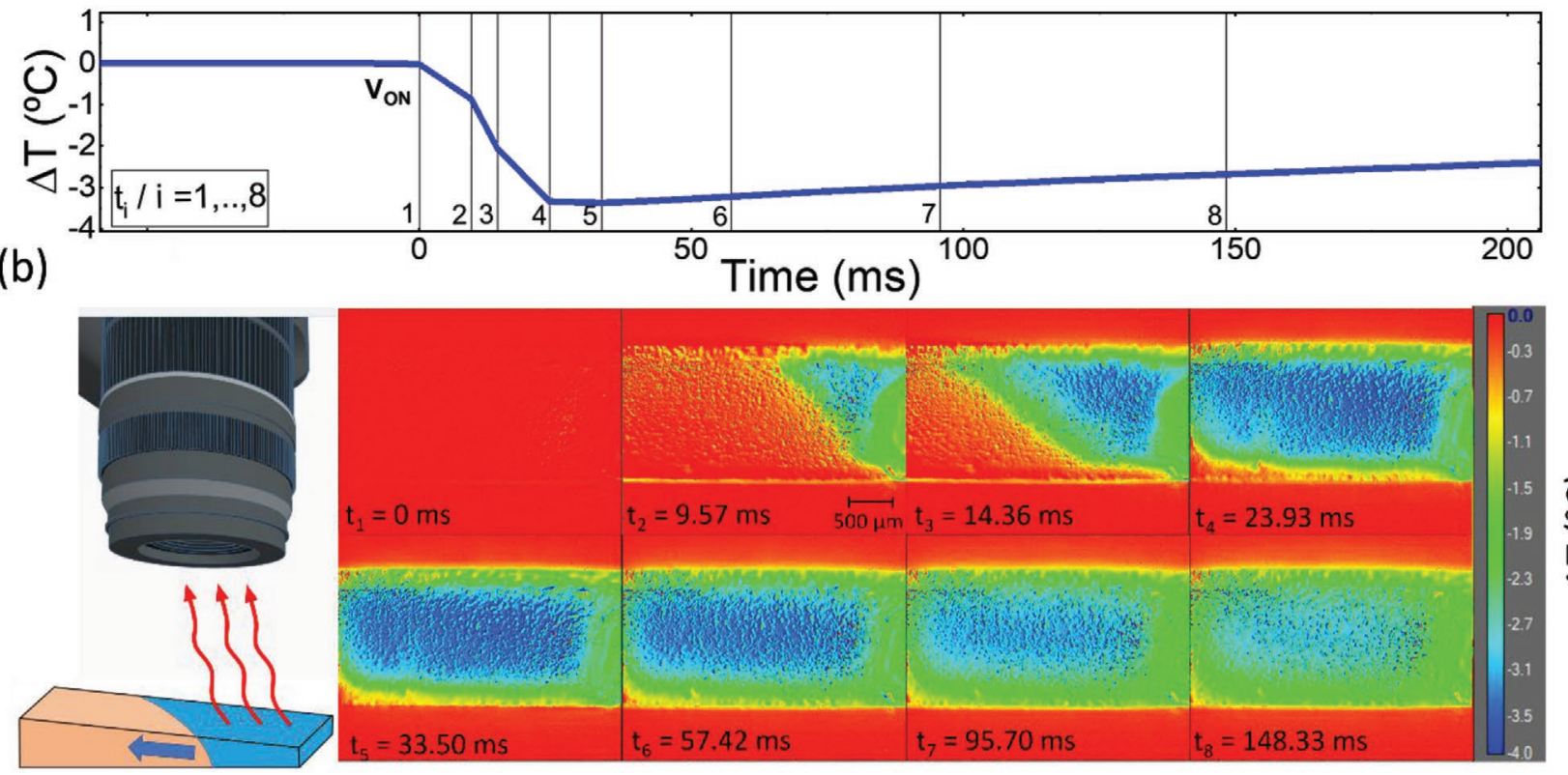

Figure 3. a) Electrocaloric profile of $\mathrm{PbZrO}_{3}$ at $T=216 \mathrm{C}$ when an average electric field of $E=35 \mathrm{kV} \mathrm{cm}^{-1}$ is applied. b) AFE-FE phase boundary propagating across the sample generating the negative ECE. The thickness gradient creates a sideways propagation of the phase boundary. Each frame has dimensions of $3.2 \mathrm{~mm} \times 2.55 \mathrm{~mm}$. Note: Pt electrodes do not reach the edges of the sample (to avoid air breakdown between the capacitor faces). This is the reason of the $\Delta T$ degradation at the edges, as no effective electric field is being applied there.

sites that appear as "cold spots" within less than $1 \mathrm{~ms}$ and full device switch in $\approx 7.9 \mathrm{~ms}$ with a concomitant $\Delta T=-3.2^{\circ} \mathrm{C}$.

In contrast, samples with thickness gradients can be used to concentrate the nucleation at one side of the sample and cause a sideways propagation of the thermal front, which may be useful for guiding the flow of heat (heat pumping or phononic guiding). Meanwhile, gradients of temperature can be used to bridge the phase diagram of $\mathrm{PbZrO}_{3}$, so that application of voltage causes one side to get colder while the other gets warmer (Janus electrocaloric effect). The results emphasize the importance of anti-ferroelectric switching dynamics for electrocaloric device performance, as well as the exciting possibilities brought to bear by the combination of in situ observation and gradient manipulation. (a)

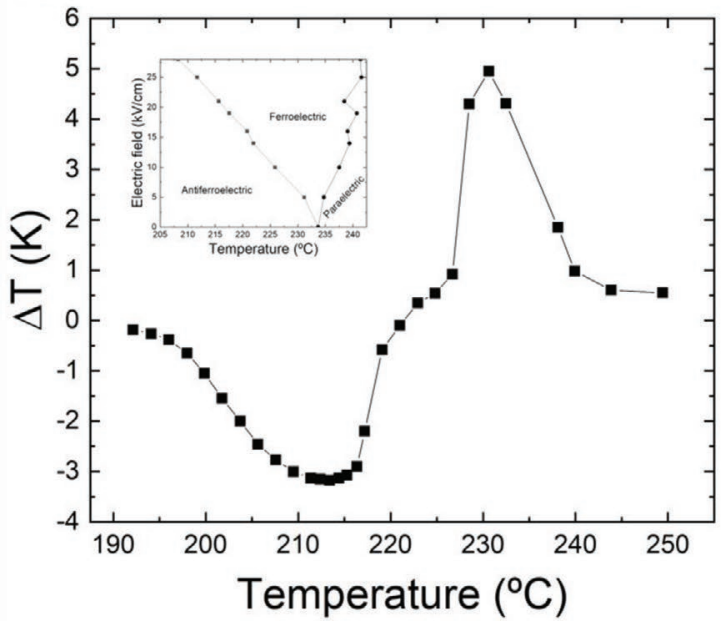

(b)

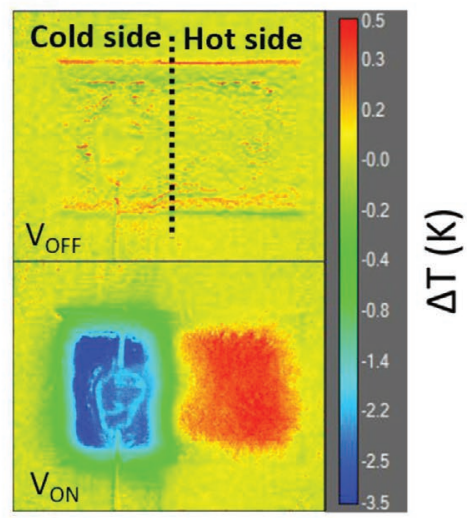

Figure 4. a) Electrocaloric profile of $\mathrm{PbZrO}_{3}$ ceramics as a function of temperature at $E=35 \mathrm{kV} \mathrm{cm}^{-1}$. Inset represents a simplified phase diagram modified from ref. [6]. b) Realization of a Janus-like behavior whereby the sample displays simultaneously a negative and a positive electrocaloric effect by setting both sides of the sample at different temperatures, namely $T=214^{\circ} \mathrm{C}$ and $T=223^{\circ} \mathrm{C}$ for the cold and hot sides, respectively. Frame dimensions: $3.2 \mathrm{~mm} \times 2.55 \mathrm{~mm}$. Note that the temperature bar represents a relative measure. 


\section{Experimental Section}

Fabrication and Sample Preparation: The electrocaloric effect of ceramics of the perovskite anti-ferroelectric archetype, $\mathrm{PbZrO}_{3}$, was measured. The samples were made as described in ref. [31] (average grain size of $5 \mu \mathrm{m}$ ), and the disk was polished down to thicknesses between 100 and $150 \mu \mathrm{m}$ with a Multiprep polishing system with a final surface roughness of $1 \mu \mathrm{m}$. Samples were not subjected to any thermal treatment afterward. Energy-dispersive X-ray spectroscopy (EDX) measurements showed that the $\mathrm{Pb} / \mathrm{Zr}$ ratio was homogeneous across the sample with a slight $\mathrm{Pb}$ deficiency of $3.5 \% \pm 0.96 \% .200 \mathrm{~nm}$ thick platinum electrodes were deposited by electron beam evaporation, and platinum wires were bonded with curated silver paste.

Infrared Characterization: The electrocaloric dynamics was measured by IR thermometry, using two different infrared cameras: an FLIR x6580sc and an FLIR SC5500. For the former, the acquisition speed used was $130 \mathrm{fps}$ while for the latter, it was 376 or $1253 \mathrm{fps}$. The lens was a $\mathrm{G} 3$ with a field of view (FOV) of $3.2 \mathrm{~mm} \times 2.56 \mathrm{~mm}$ and $10 \mu \mathrm{m}$ lateral resolution. Prior to the IR characterization, the samples were covered with an emissivitycalibrated black paint and located in a Linkam chamber (model T95-PE), based on a Peltier temperature control, coupled to a micropositioning system to allow their correct focusing, displacement, and setting their initial temperature. The electrocaloric effect was induced by a Keithley 2410 High Voltage Sourcemeter with voltage rise times of $300 \mu \mathrm{s}$. The measuring process is based on the dynamics of a Brayton cycle; applying a voltage step adiabatically acquires the response and lets the sample thermalize before adiabatically removing the field. The relative temperature changes acquired with the IR camera were measured with an accuracy of $0.1 \mathrm{~K}$.

\section{Acknowledgements}

The authors acknowledge financial support to ICN2, which is funded by the CERCA programme/Generalitat de Catalunya and by the Severo Ochoa programme of the Spanish Ministry of Economy, Industry and Competitiveness (MINECO, Grant No. SEV-2017-0706). The authors also acknowledge the support of Plan Nacional (MINECO, Grant Nos. MAT2016-77100-C2-1-P and BES-2016-077392), as well as the Agencia Estatal de Investigacion (Grant No. PID2019-108573GB-C21). R.F. and E.D. thank the Luxembourg National Research Fund (FNR) for funding part of this research through the projects CAMELHEAT/ C17/MS/11703691/Defay. This work was also supported in part by the Spanish Ministry of Science, Innovation and Universities under the HIPERCELLS project (RTI2018-098392-B-I00), the Regional Government of the Generalitat de Catalunya under Grant Nos. 2017 SGR 1384 and 2017 SGR 00579. This work was also supported by the National Science Centre, Poland, within the Project No. 2016/21/B/ST3/02242.

\section{Conflict of Interest}

The authors declare no conflict of interest.

\section{Data Availability Statement}

Research data are not shared.

\section{Keywords}

anti-ferroelectrics, electrocalorics, infrared thermometry, phase boundary

Received: May 3, 2021 Published online:
[1] Q. Correia, T. Zhang, Electrocaloric Materials: New Generation of Coolers, Springer Verlag, Berlin 2014.

[2] T. Reid, Philos. Mag. 1878, 5, 4.

[3] A. Mischenko, Q. Zhang, J. F. Scott, R. W. Whatmore, N. D. Mathur, Science 2006, 311, 1270

[4] W. Geng, Y. Liu, X. Meng, L. Bellaiche, J. F. Scott, B. Dkhil, A. Jiang, Adv. Mater. 2015, 27, 3165.

[5] R. Pirc, B. Rožič, J. Koruza, B. Malič, Z. Kutnjak, EPL 2014, 107, 17002.

[6] P. Vales-castro, R. Faye, M. Vellvehi, Y. Nouchokgwe, X. Perpiñà, J. M. Caicedo, X. Jordà, K. Roleder, D. Kajewski, A. Perez-Tomas, E. Defay, G. Catalan, Phys. Rev. B 2021, 103, 54112.

[7] A. Lubk, S. Gemming, N. A. Spaldin, Phys. Rev. B: Condens. Matter Mater. Phys. 2009, 80, 104110.

[8] W. J. Merz, J. Appl. Phys. 1956, 27, 938.

[9] W. J. Merz, Phys. Rev. 1954, 95, 690.

[10] R. C. Miller, A. Savage, Phys. Rev. 1958, 112, 755.

[11] R. C. Miller, G. Weinreich, Phys. Rev. 1960, 117, 1460.

[12] C. T. Nelson, P. Gao, J. R. Jokisaari, C. Heikes, C. Adamo, A. Melville, S.-H. Baek, C. M. Folkman, B. Winchester, Y. Gu, Y. Liu, K. Zhang, E. Wang, J. Li, L.-Q. Chen, C.-B. Eom, D. G. Schlom, X. Pan, Science 2011, 334, 968.

[13] J. F. Scott, in Ferroelectric Memories, Springer Series in Advanced Microelectronics (Eds: K. Itoh, T. Sakurai), Springer-Verlag, Berlin, Heidelberg, New York 2000, p. 3.

[14] J. Y. Jo, D. J. Kim, K. Sun-Young, S.-B. Choe, T. K. Song, J.-G. Yoon, T. W. Noh, in 2007 16th IEEE Int. Symp. on the Applications of Ferroelectrics, IEEE, Piscataway, NJ 2007, pp. 28-31. https://doi. org/10.1109/ISAF.2007.4393157.

[15] H. L. Stadler, P. J. Zachmanidis, J. Appl. Phys. 1963, 34, 3255.

[16] A. S. Sidorkin, L. P. Nesterenko, B. M. Darinskii, A. A. Sidorkin, S. V. Senkevich, E. V. Vorotnikov, N. H. Thuong, Mater. Res. Bull. 2017, 96, 206.

[17] A. Y. Woldman, C. M. Landis, Smart Mater. Struct. 2016, 25, 035033.

[18] M. DiDomenico, S. H. Wemple, Phys. Rev. 1967, 155, 539.

[19] V. M. Ishchuk, Ferroelectrics 2001, 254, 275.

[20] Y. Li, Q. Yan, Y. Zhang, X. Xi, X. Chu, W. Cao, Appl. Phys. Lett. 2012, 101, 132904

[21] S. Puchberger, V. Soprunyuk, A. Majchrowski, K. Roleder, W. Schranz, Phys. Rev. B 2016, 94, 214101.

[22] B. Liu, X. Tian, L. Zhou, X. Tan, Phys. Rev. Mater. 2020, 4, 104417.

[23] M. Si, X. Lyu, P. R. Shrestha, X. Sun, H. Wang, K. P. Cheung, P. D. Ye, Appl. Phys. Lett. 2019, 115, 072107.

[24] X. Moya, E. Stern-Taulats, S. Crossley, D. González-Alonso, S. Kar-Narayan, A. Planes, L. Mañosa, N. D. Mathur, Adv. Mater. 2013, 25, 1360.

[25] D. Guo, J. Gao, Y.-J. Yu, S. Santhanam, G. K. Fedder, A. J. H. McGaughey, S. C. Yao, Appl. Phys. Lett. 2014, 105, 031906.

[26] Y. Liu, B. Dkhil, E. Defay, ACS Energy Lett. 2016, 1, 521.

[27] A. V. Kimmel, O. T. Gindele, D. M. Duffy, R. E. Cohen, Appl. Phys. Lett. 2019, 115, 023902.

[28] M. Guo, M. Wu, W. Gao, B. Sun, X. Lou, J. Mater. Chem. C 2019, 7, 617.

[29] S. Crossley, Ph.D. Thesis, University of Cambridge 2013.

[30] K. M. Rabe, in Functional Metal Oxides: New Science and Novel Applications (Eds: S. B. Ogale, T. V. Venkatesan, M. G. Blamire), Wiley-VCH, Weinheim, Germany 2013, pp. 221-244.

[31] K. Roleder, K. Wojcik, Ferroelectrics 1984, 61, 293. 